\title{
A manifesto: circus Island
}

\section{Alvado Bañón, Joaquín ${ }^{1}$}

${ }^{1}$ Department of Graphic Expression, Design and Projects / Alicante University, Spain joaquin.alvado4@gmail.com

Citation: Alvado Bañón, J. (2021). A Manifesto: Circus Island. UOU scientific journal \#01, 22-25.

ISSN: 2697-1518. https://doi.org/10.14198/UOU.2021.1.03

This document is under a Creative Commons Attribution 4.0 International license (CC BY 4.0)

First, we need an Architectonical space for learning. We name it. Circus Island.

Circus Island must be a constellation of public spheres as narratives of autonomous subjectivity. We will seek to enact a multidisciplinary direction through which architectonic practices and processes come most alive. We will constitute a map of contemporary knowledge circuits where art, theory, science, culture, ecology, and politics collide. Urban systems and metaterritory experience open to freedom openness and changes. No verb in this sentence.

UOU, our international studio, is an area of uncertainty and reflection. This space must be built to embrace everyone; students, teachers, institutions, guests and citizens, hundreds of voices within a discursive dynamic system.

UOU, the "Circus Island" is a democratic space for discussion.

The construction of this experimental space need:

1. Openness. To incorporate open formats and new work contexts, experimental practices and learning systems.

2. To incorporate student's upcoming and active vision. The student is part of our teaching. Our goal is autonomy based on their own maps of projective action.

3. To embrace diversity. Working on broad contexts. Students and teachers experience on their capabilities, knowledge and limits as an essential part for the teaching portfolio.

4 Proposals for the future. The space is for managing the future reality.

5. Uncertainty. Emotion to work on the unknown, to find the 
structure for the fiction. To accept complexity of knowledge in contemporary teaching.

6. Research as autonomy of thought. To be committed to scientific knowledge to expand research and knowledge.

7. Imagination. Imaginative space as a process of relationship.

8. To build an exhibition space, a document. UOU is a document, a portfolio in itself.

9. Discussion as a search for knowledge. To accept the multidisciplinary approach of a changing profession.

11. $\underline{\text { Risk. }}$. The UOU was born in an unreferenced context, on a bizarre online periphery.

12. Dynamicity. Travel for deferred knowledge.

The Circus Island; the space for UOU, is a collective, dynamic, experiential and future-looking process. It is for living and to live in. It is productive and exciting. It is an adventure, a risk.

Circus Island: A leisure space, a space for fun, to enjoy and to spend a good time, a space for happiness; a soft space, a space to love, to fraternize, to become close friends; a conflictive space, a space to argue, to discuss and to win.

\section{MASK}

"Architecture is a fuzzy amalgamation of ancient knowledge and contemporary practice, an awkward way to look at the world and an inadequate medium to operate on it... Architecture is too slow. Yet, the word "architecture" is still pronounced with certain reverence (outside of the profession). It embodies the lingering hope -or the vague memory of hope- that shape, form, coherence could be imposed on the violent surf of information that washes over us daily. Maybe, architecture doesn't have to be stupid after all. Liberated from the obligation to construct, it can become a way of thinking about anything -a discipline that represents relationships, proportions, connections, effects, and the diagram of everything."

Rem Koolhass. "Content"

Circus Island is a critical and experimental vision of contemporary urban society. We propose a Mask; an architecture about the making of architecture.

Masking ourselves as a process in which an individual changes or "masks" their natural personality. We will work to find our architectonic mask and build it. We will become architects.

As Hejduk in Victims; We will project our Island to portray an analogous city, an experimental version of the contemporary city.

Radicality, detour, digression as critical reflection.

Architectural typology, to mobilize a repertoire of building elements drawn into an exchange between theatrical and formal/expressive practices. 


\section{LANGUAGE}

Language systems reside in individual minds, they have a separate existence and thus remain detached from their users.

Rorty defines an "ironist as someone who fulfills three conditions.

(1) She has radical and continuing doubts about the final vocabulary she currently uses, because she has been impressed by other vocabularies, vocabularies taken as final by people or books she has encountered.

(2) She realizes that argument phrased in her present vocabulary can neither underwrite nor dissolve these doubts.

(3) Insofar as she philosophizes about her situation, she does not think that her vocabulary is closer to reality than others, that it is in touch with a power not herself.

Ironists describe themselves as subject to change, always aware of the contingency and fragility of their final vocabularies, and thus of themselves.

The opposite of irony is common sense. UOU needs a new vocabulary to establish the rules of our architecture. Let us find a foolish act or idea

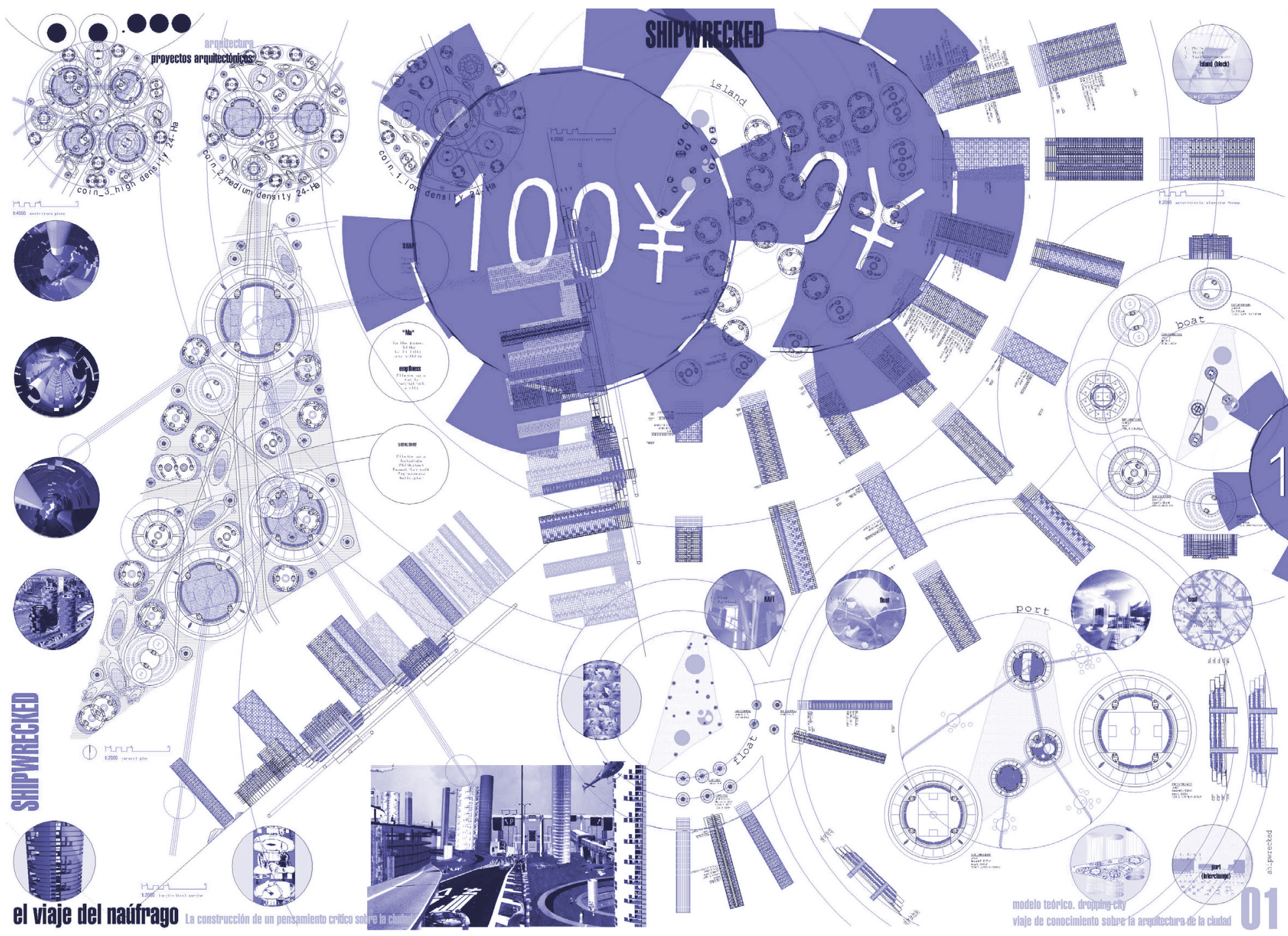




\section{DYNAMIC KNOWLEDGE}

In Circus Island we try to find the language throw the vocabulary. What do we refer to when we talk about Architecture?

This is the question for our architectural research at the UOU. We want to talk from scratch about innovation, social behaviour, nature, growth, time, change, sustainability, weather, citizenship, atmosphere, water, adaptability, welfare and environment. We use research as a technical tool to work on diversity, an open future, emotion, imagination and a complete portfolio to establish a dynamic process that will help us formulate concepts concerning architecture and life.

Dynamic knowledge is related to time, space and existence. The online procedures are real dynamic systems, chaotic descriptions. In dynamic systems, time and space are interdependent; there is no apparent center or order. In our research, we focus on dynamic grids of geometry, which create the shape of a structure, thus giving architectural design presence. As a Dynamic system, we work with iteration and sensitivity as initial conditions. It is under those parameters that a system process becomes a structure. Our approach is related to two main items: emptiness and dynamic systems. Emptiness is related to iteration and recursion, while dynamic systems are related to sensitivity to initial conditions.

Emptiness as a "democratic space" is projected as a conflict between virtual and real space. It gives architecture a kind of depth that is not exhausted on a spatial scale, but works jointly with virtual space to manifest its void as a possibility. It is a probability, and it is shown as a domain within the realm of possibility. It transcends architecture's perception as an "object" at the moment or stage that we become aware of its existence. Networks connecting spaces are instruments for understanding their structural functions. Diagrams and grid maps depend on the network shape and quality. In our research, networks are based on cultural background. New policies for sustainable cultural thought will become the answer to future design questions and new identity systems.

Our approach works with dynamic, online structures. Each unit becomes a tensional network of visual negotiations. Virtuality reinforces the effect of chaotic order in such a way that certain "informal" elements become structural supports for space. The visual complex of spatial interconnections is established through democratic architecture as an emptiness. The tensional organisation is based on grids, on creating three-dimensional connections in which order is magnified into a labyrinth of structures at a small-object scale.

Circus Island is a dynamic system. Dynamic systems' sensitivity to initial conditions means that a system's asymptotic behaviour can be altered by even the slightest of changes in the initial conditions: "the butterfly effect". As we well know, we need to educate the next generations to stay true to the initial conditions. 